\title{
Investigation of Band Gap Width in Ternary 1D Photonic Crystal with Left-Handed Layer
}

\author{
Z. Zare AND A. GharaAti* \\ Department of Physics, Payame Noor University, I.R. of Iran \\ (Received January 6, 2013; in final form October 20, 2013)
}

\begin{abstract}
In this paper, we investigate the variation of band gap width in a ternary 1D photonic crystal with a left-handed layer versus variation of thicknesses and refractive index of second layers. We study these variations in two states, normal and oblique incidents for both TE and TM polarizations. We observe that the variations of band gap width versus thickness of layers are sensitive to the refractive index of second layers. For oblique incident both of variations are shifted towards the large/small frequencies by increasing the incident angles in TE/TM polarization, respectively.
\end{abstract}

DOI: 10.12693 /APhysPolA.125.36

PACS 42.70.Qs

\section{Introduction}

Photonic crystals (PCs) are structures with modulation of the refractive index and they have received much attention for their interest applications $[1,2]$. PCs have many interesting applications such as omnidirectional reflector [3], filters, optical switches, light-emitting diodes [4], fibers [5], waveguides [6] etc. When the light wave is incident on PCs, they face with number of dielectric layers with different dielectric constants [7]. These differences between each two adjacent layers cause reflectance and transmittance from their interface and the interface between the reflected and transmitted light wave appears in form of photonic band gaps. These band gaps are in the range of frequency that the electromagnetic waves cannot propagate in them $[8,9]$.

Left-handed materials (LHM) are artificial structures with simultaneously negative permittivity $\varepsilon$ and negative permeability $\mu$ [10-12]. When an electromagnetic wave propagates through these structures, the direction of the Poynting vector is opposite to the wave vector $\boldsymbol{k}[9]$. Here we study the variation of a ternary $1 \mathrm{D}$ PC with a left-handed layer versus the thicknesses and refractive index of layers in normal and oblique incidents.

\section{Theory}

Consider a monochromatic wave incident on a ternary 1D PC as we have shown in Fig. 1. Each unit cell consists of 3 layers $n_{1}>0, n_{2}<0$, and $n_{3}>0$. Assume that $x-z$ is the incident plane and $z$ is the periodic axis, so the refractive index in the unit cells varies as follows:

$$
n(z)= \begin{cases}n_{1}, & 0<z<d_{1}, \\ n_{2}, & d_{1}<z<d_{2}, \\ n_{3}, & d_{2}<z<\Lambda .\end{cases}
$$

\footnotetext{
*corresponding author; e-mail: agharaati@pnu.ac.ir
}

Here $n_{i}, i=1,2,3$ are the refractive indices, $d_{i}$ is the thickness of each layer and $\Lambda=d_{1}+d_{2}+d_{3}$ is the period of structure.

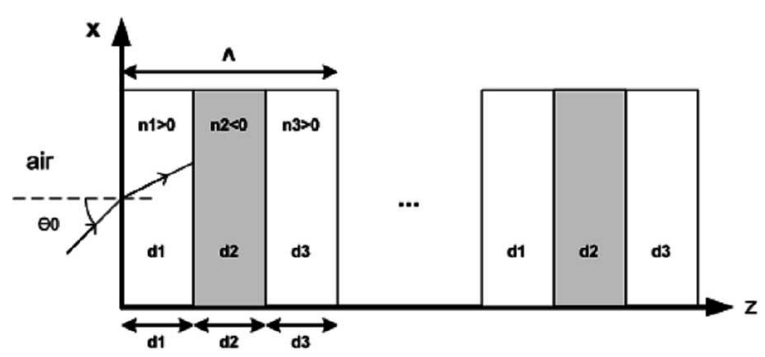

Fig. 1. The structure of a ternary 1D photonic crystal with a left-handed layer.

The electric field in each layer of unit cell can be written as

$$
\begin{aligned}
& E_{1 y}=A_{1} \mathrm{e}^{\mathrm{i} k_{1} z}+B_{1} \mathrm{e}^{-\mathrm{i} k_{1} z}, \\
& E_{2 y}=C_{1} \mathrm{e}^{\mathrm{i} k_{2}\left(z-d_{1}\right)}+D_{1} \mathrm{e}^{-\mathrm{i} k_{2}\left(z-d_{1}\right)}, \\
& E_{3 y}=E_{1} \mathrm{e}^{\mathrm{i} k_{3}\left(z-\left(d_{1}+d_{2}\right)\right)}+F_{1} \mathrm{e}^{-\mathrm{i} k_{3}\left(z-\left(d_{1}+d_{2}\right)\right)} .
\end{aligned}
$$

In these relations $A_{1}, C_{1}$, and $E_{1}$ are the amplitudes of the incident wave and $B_{1}, D_{1}$, and $F_{1}$ are the amplitudes of the reflective wave in first, second, and third layer, respectively.

$$
\begin{aligned}
k_{i} & =\left[\left(\frac{n_{i} \omega}{c}\right)^{2}-\beta_{i}^{2}\right]^{1 / 2}, \quad i=1,2,3, \\
\beta_{i} & =\frac{n_{i} \omega}{c} \sin \theta_{i}
\end{aligned}
$$

is the wave number along $x$ axis. $n_{i}=\sqrt{\varepsilon_{i} \mu_{i}} i=1,3$ are refractive indices of first and third layers and $n_{2}=$ $-\sqrt{\varepsilon_{2} \mu_{2}}$ is refractive index of the second layer. $\varepsilon_{i}$ and $\mu_{i}$ are the permittivity and permeability of each layer, respectively. Using the continuity conditions, we can obtain the transfer matrix of each layer of unit cell as [9]: 


$$
M_{j}=\frac{1}{2}\left[\begin{array}{ll}
\left(1+\delta_{j}\right) \mathrm{e}^{\mathrm{i} \varphi_{j}} & \left(1-\delta_{j}\right) \mathrm{e}^{-\mathrm{i} \varphi_{j}} \\
\left(1+\delta_{j}\right) \mathrm{e}^{\mathrm{i} \varphi_{j}} & \left(1-\delta_{j}\right) \mathrm{e}^{-\mathrm{i} \varphi_{j}}
\end{array}\right], \quad j=1,2,3,
$$

where $\delta_{1}=\frac{k_{1} \mu_{2}}{k_{2} \mu_{1}}, \delta_{2}=\frac{k_{2} \mu_{3}}{k_{3} \mu_{2}}$, and $\delta_{3}=\frac{k_{3} \mu_{1}}{k_{1} \mu_{3}}$ for TE polarization. For TM polarization these relations are as the same but we just replace $\varepsilon$ with $\mu . \varphi_{j}=k_{j} d_{j}$ is the phase of each medium.

The transfer matrix of unit cell is given by [13]:

$$
M_{0}=M_{3} M_{2} M_{1} \text {. }
$$

If all unit cells were identical and by assuming the system is lossless, the total transfer matrix is

$$
M=M_{0}^{N} \text {. }
$$

Using the Bloch theorem $[8,14]$ and transfer matrix we can obtain the dispersion relation as

$$
k(\omega)=\frac{1}{\Lambda} \arccos \left(\frac{1}{2}\left(M_{0}(1,1)+M_{0}(2,2)\right)\right) .
$$

Regimes in which $\left|(1 / 2)\left(M_{0}(1,1)+M_{0}(2,2)\right)\right|<1$, are related to the propagating Bloch waves, when $\left|(1 / 2)\left(M_{0}(1,1)+M_{0}(2,2)\right)\right|>1, k$ is imaginary so that the Bloch wave is evanescent. These are forbidden bands. Therefore, the band edges are obtained by solving the equation $\left|(1 / 2)\left(M_{0}(1,1)+M_{0}(2,2)\right)\right|=1$. For solving this equation, we should obtain the roots of right hand side of the equation with the line $y=1$ and then getting the difference of two consecutive roots.

\section{Variation of BGW by changing the thickness of layers}

In this section, we plot the variation of the first and second BGW by changing the thickness of second and third layer at different refractive index of second layer [15]. The wall between these layers are moved such that $d_{2}+d_{3}=$ const (Fig. 2).

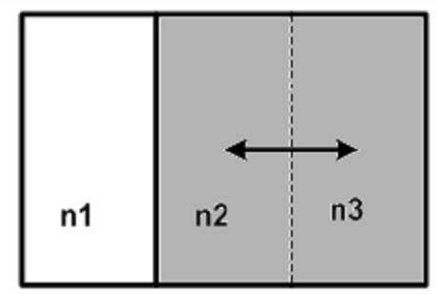

Fig. 2. The variation of thickness of the second and third layers in a ternary $1 \mathrm{D}$ PC with left-handed layer.

Figure 3 shows these variations for the first and second BGW structure with $n_{1}=1, n_{3}=3.5, \mu_{1}=\mu_{3}=1$, $\mu_{2}=-1, d_{1}=1 \mu \mathrm{m}, d_{2}+d_{3}=1 \mu \mathrm{m}$. The number of unit cells are $N=10$ and $\theta_{0}=0$. In all cases the BGWs are normalized to the Bragg frequency, $v_{\mathrm{B}}=(c / \bar{n}) / 2 \Lambda$, in which $\bar{n}=\left(n_{1} d_{1}+n_{2} d_{2}+n_{3} d_{3}\right) / \Lambda$ is the average refractive index of PC.

As we observe from Fig. 3, these variations are sensitive to the refractive index of second layer and by changing its own magnitude they are shifted towards the larger

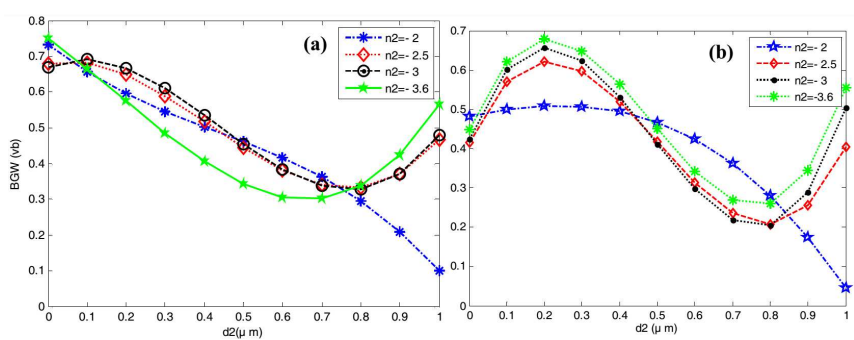

Fig. 3. The variation of (a) the first, (b) the second BGW versus the thickness of the second layer at different refractive index for the structure with left-handed layer.

BGWs. In addition, variations trends of all mentioned refractive indices are the same except for $n_{2}=-2$.

\section{Variations of BGW by changing the refractive index of left-handed layer}

In this section, we consider the structure with characteristics of $n_{1}=1, n_{2}=-2$ to $-3, n_{3}=3.5, \mu_{1}=\mu_{3}=1$, $\mu_{2}=-1, \theta_{0}=0$ and $N=10$. Then, we plot the variation of first and second BGW versus the refractive index of second layer in Fig. 4.

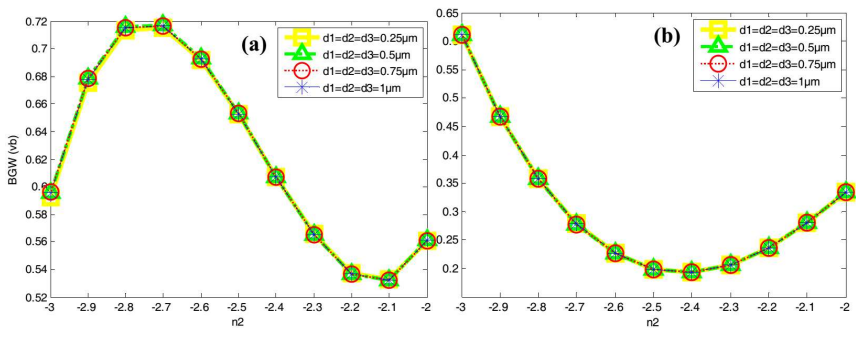

Fig. 4. Variation of (a) the first, (b) the second band gap in the structure with left-handed layer for different thickness of layers.

From Fig. 4, we conclude that the variations of BGW with different thickness of layers are completely compatible and overlap on each other.

\section{Variations of BGW by changing the refractive index of second layer at different incident angles}

We show the variation of BGW for the structure with $n_{1}=1, n_{2}=-2$ to $-3, \mu_{1}=\mu_{3}=1, \mu_{2}=-1, d_{1}=$ $d_{2}=d_{3}=1.6 \mu \mathrm{m}, N=10$ and for incident angles 0 , 30, 45, 60, and 70 degrees for TE polarization in Fig. 5a and TM polarization in Fig. 5b. As shown in Fig. 5a, the BGWs are shifted towards large magnitudes as the incident angles are increased. These variations are more sensitive around more negative refractive indices. For Fig. 5b these variations are more complex but the trend of them is getting decreased as the angle of the incidence is increased. 


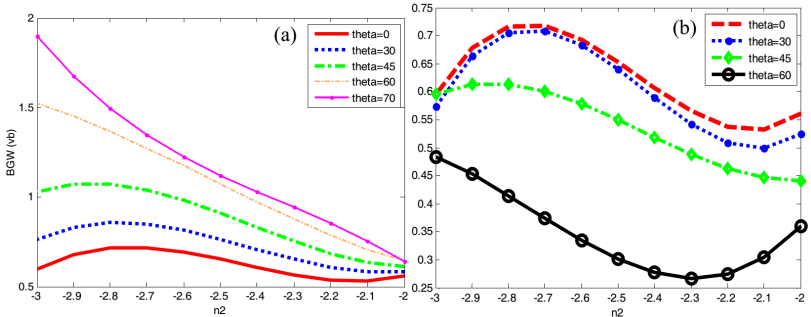

Fig. 5. Variation of the first BGW versus refractive index of second layer at different incident angles: (a) TE polarization, (b) TM polarization.

\section{Variations of BGW by changing the thickness of second and third layer in different incident angles}

In this part, we consider a PC with $n_{1}=1, n_{2}=-2$, $n_{3}=3.5, \mu_{1}=\mu_{3}=1, \mu_{2}=-5, d_{1}=1 \mu \mathrm{m}, d_{2}+d_{3}=$ $1 \mu \mathrm{m}$ and $N=10$. Then, we plot the variation of BGW for this structure under the different incident angles at TE and TM polarizations in Fig. 6.

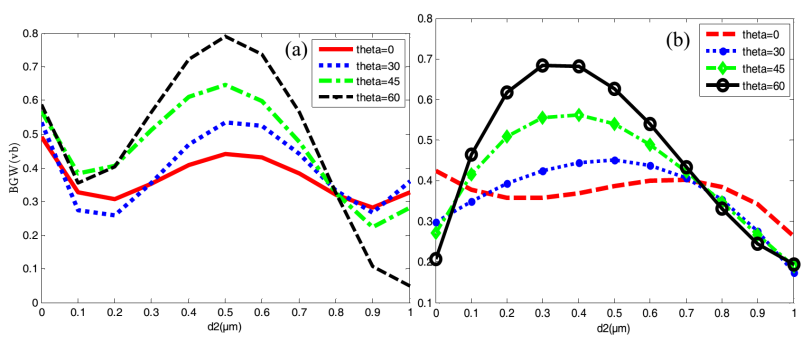

Fig. 6. Variation of first band gap in a ternary PC with a left-handed layer versus thickness of second layer at different incident angles: (a) TE, (b) TM polarizations.

As shown in Fig. 6a, all the diagrams have a maximum that goes up as the incident angle is increased and all of them have approximately the same trend. For TM polarization, the variation trend is different for $\theta=0$ but it is the same for other angles. In this case the maximum of the diagrams goes up as the angle is increased as well.

\section{Conclusion}

By using the transfer matrix method, we could obtain the variation of the $B G W$ versus refractive index and thicknesses of layers. We studied these variations in two states, normal and oblique incidents. According to our observation, these variations are sensitive to the refractive index of second layer and they are shifted towards the larger BGWs as refractive index of second layer changed. In addition, variations trends of all mentioned refractive indices are the same except for $n_{2}=-2$, while these variations versus the refractive index of second layer are completely compatible and overlap on each other at different thicknesses. For the oblique incident in the case of TE polarization, the variations versus the refractive index of second layer of the BGWs are shifted towards large magnitudes as the incident angles are increased. These variations are sensitive around more negative refractive indices. For TM polarization the variations versus refractive index are more complex but the trend of them is getting decreased as the incident angles increased. When we plot the variations of BGW versus the different thickness of second layer we noticed that all the diagrams have a maximum that goes up as the incident angle increased.

\section{Acknowledgments}

This work has been financially supported by the Payame Noor University (PNU) I.R. of Iran under the grant Dr. Abdolrasoul Gharaati.

\section{References}

[1] J.C. Knight, F. Luan, G.J. Pearce, A. Wang, T.A. Birks, D.M. Bird, Jpn. J. Appl. Phys. 45, 6059 (2006).

[2] E. Ozbay, I. Bulu, K. Guven, H. Caglayan, K. Aydin, Jpn. J. Appl. Phys. 45, 6064 (2006).

[3] A. Gharaati, Z. Zare, Prog. Electromagn. Res. M 20, 81 (2011).

[4] N. Nozhat, N. Granpayeh, Prog. Electromagn. Res. 99, 225 (2009).

[5] R. Buczynski, Acta Phys. Pol. A 106, 141 (2004).

[6] V.G. Baryshevesky, P.V. Molchanov, Acta Phys. Pol. A 115, 971 (2009).

[7] J.D. Joannopoulous, R.D. Meade, J.N. Winn, Photonic Crystals: Molding the Flow of Light, 2nd ed., Princeton University Press, New Jersey 2008.

[8] P. Yeh, A. Yariv, C.S. Hong, J. Opt. Soc. Am. 67, 423 (1976).

[9] S.K. Srivastava, S.P. Ojha, Prog. Electromagn. Res. 68, 91 (2007).

[10] S.A. Ramakrishna, Rep. Prog. Phys. 68, 449 (2005).

[11] V.G. Veselago, Sov. Phys.-Usp. 10, 509 (1968).

[12] R. Marques, F. Martin, M. Sorolla, Metamaterials with Negative Parameters: Theory, Design and Microwave Applications, Wiley Series in Microwave and Optical Engineering, New Jersey 2007.

[13] B.E.A. Saleh, M.C. Teich, Fundamentals of Photonics, 2nd ed., Wiley, New York 2007.

[14] A. Yariv, P. Yeh, Photonics Optical Electronics in Modern Communications, Oxford University Press, Hoboken (NJ) 2007.

[15] A. Gharaati, S.A. Serajfard, IEEE Xplore, 2010 Int. Conf. Photonics (ICP) ICP2010-48, 1 (2010). 